\title{
Biophysical Considerations in the Rational Design and Cellular Targeting of Flexible Polymeric Nanoparticles
}

\section{Samaneh Farokhirad}

New Jersey Institute of Technology

Sreeja Kutti Kandy

University of Pennsylvania

Andrew Tsourkas

University of Pennsylvania

Portonovo Ayyaswamy

University of California, Los Angeles

David Eckmann

Ohio State University

Ravi Radhakrishnan ( $\nabla$ rradhak@seas.upenn.edu )

University of Pennsylvania https://orcid.org/0000-0003-0686-2851

\section{Article}

Keywords: nanoparticles (NPs), flexibility, engineered physicochemical characteristics

Posted Date: May 24th, 2021

DOI: https://doi.org/10.21203/rs.3.rs-477842/v2

License: (9) (i) This work is licensed under a Creative Commons Attribution 4.0 International License. Read Full License

Version of Record: A version of this preprint was published at Advanced Materials Interfaces on November 11th, 2021. See the published version at https://doi.org/10.1002/admi.202101290. 


\title{
Biophysical Considerations in the Rational Design and Cellular Targeting of Flexible Polymeric Nanoparticles
}

\author{
Samaneh Farokhirad ${ }^{1,2},{ }^{*}$ Sreeja Kutti Kandy ${ }^{2},{ }^{+}$Andrew Tsourkas ${ }^{3,},{ }^{\ddagger}$ Portonovo \\ S. Ayyaswamy ${ }^{4,6}$, Savid M. Eckmann ${ }^{5}$, " and Ravi Radhakrishnan ${ }^{2,3 * *}$ \\ ${ }^{1}$ Department of Mechanical Engineering, New Jersey Institute of Technology, Newark, NJ, USA \\ ${ }^{2}$ Department of Chemical and Biomolecular Engineering, University of Pennsylvania, Philadelphia, PA, USA \\ ${ }^{3}$ Department of Bioengineering, University of Pennsylvania, Philadelphia, PA, USA \\ ${ }^{4}$ Department of Mechanical Engineering and Applied Mechanics, University of Pennsylvania, Philadelphia, \\ $P A, U S A$ \\ ${ }^{5}$ Department of Anesthesiology, The Ohio State University, Columbus, $\mathrm{OH}, \mathrm{USA}$ \\ ${ }^{6}$ Department of Mechanical and Aerospace Engineering, University of California, Los Angeles, CA, USA
}

\begin{abstract}
Physicochemical characteristics of nanoparticles (NPs) can be engineered for tuning their biological function in cellular delivery. How NP mechanical properties impact multivalent ligand-receptor mediated binding to cell surfaces, the avidity of NP adhesion to cells, propensity for internalization, and effects due to crowding remain unknown or unquantified. We report computational analyses of binding mechanisms of three distinct NPs that differ in type and rigidity (core-corona flexible NP, rigid NP, and rigid-tethered NP) but are otherwise similar in size and ligand surface density; moreover, for the case of flexible NP, we tune NP stiffness by varying the internal crosslinking density. We employ biophysical modeling of NP binding to membranes together with thermodynamic analysis powered by free energy calculations and show that efficient cellular targeting and uptake of NP functionalized with targeting ligand molecules can be shaped by factors including NP flexibility and crowding, receptor-ligand binding avidity, state of the membrane cytoskeleton, and curvature inducing proteins. Owing to this multitude of factors, we demonstrate that the binding avidity of a flexible NP depends on engineered changes in NP flexibility governed by significant enthalpy entropy compensations
\end{abstract}


arising from multiple competing terms associated with NP, receptor density, and membrane. Analyses of the individual enthalpic and entropic contributions associated with NP, membrane, and receptor-ligand binding and receptor diffusion collectively illuminate this complex dependence of avidity on crosslinking. These findings provide strong evidence that NP flexibility is an important design parameter for rationally engineering NP targeting and uptake in a crowded cellular adhesion microenvironment.

\section{Significance Statement (120 words)}

Functional nanoparticles are finding applications in genome editing, immune modulation, and cell therapies. However, their design, optimization, and deployment are hampered by our lack of understanding of how their physicochemical characteristics inform the cellular targeting. Through computational analyses we address the effects of NP composition from soft, deformable NPs to rigid spheres, effect of tethers, the crowding of NPs at the membrane surface, and the cell membrane properties such cytoskeletal interactions. We uncover rational design principles that confer tension, membrane excess area, and cytoskeletal sensing properties to the NP which can be exploited for cell specific targeting of NP. Design principles informed through our studies open up avenues for rationally engineering and tuning of new systems in targeted drug delivery.

* samaneh.farokhirad@njit.edu

† sreeja@seas.upenn.edu

‡ atsourk@seas.upenn.edu

$\S$ ayya@seas.upenn.edu

ๆ David.Eckmann@uphs.upenn.edu

**rradhak@seas.upenn.edu 


\section{Introduction}

An understanding of the interaction of nanoparticles (NPs) with a biological cell membrane is crucial for the establishment of advanced therapeutics such as cancer immunotherapy and gene therapy ${ }^{1-3}$ and their application as biomarkers for imaging and detection ${ }^{4-6}$. Functional nanoparticles are finding applications in genome editing, immune modulation, cell therapies, and molecular diagnostics to stratify patients based on biomarkers. However, the functional NPs' design, optimization, and deployment are hampered by our lack of understanding of their biological barriers and pharmacokinetics ${ }^{7}$. While such barriers have contributions that are systemic and span multiple scales, the specificity for a given application has essential contributions from the cellular scale. Cellular targeting is governed by avidity (adhesion) and uptake (internalization), which depend on the properties of the NPs and the cellular microenvironment.

Adhesion between an NP and a cell surface typically involves the simultaneous binding ${ }^{8,9}$ of many hundreds of ligands on the NP's surface to a similar number of receptors on the cell surface ${ }^{9-13}$. The physiological outcome of such multivalent adhesion, which can mediate diverse phenomena, including cell crowding and cell uptake ${ }^{14}$, depends on the strength of ligand-receptor binding interactions. Other factors that can modulate multivalent adhesion strength include physiochemical properties of both the NPs and the cell surfaces ${ }^{15}$. Previous investigations have shown that the NP's shape and size are critical design parameters for the interaction of the particles with the membranes. However, in addition to controlling the external particle shape, it is also essential to consider how the internal structure affects the avidity ${ }^{16-18}$, in addition to the receptor-ligand binding strength. With this perspective, we consider a new class of crosslinked 
polymeric NPs called nanogel particles (NGPs), making them deformable and elastic. We mainly focus on a biocompatible NGP comprising a lysozyme-rich core with dextran brushes capable of hosting guest molecules, including small hydrophobic drugs and contrast imaging agents ${ }^{19,20}$. Furthermore, when these NPs are bound to the cell surface, they undergo deformation ${ }^{21}$. Studies in culture and in vivo have provided significant evidence for the relationship between the mechanical properties of NGPs and the enhanced targeting of NGPs to topologies that are entirely inaccessible to their counterpart rigid particles ${ }^{22}$. The extent of the deformation of NGPs may then be controlled via either tuning the polymer crosslinking density (i.e., elasticity) or impacted by the cell surface mechanical properties. The crosslinked polymer networks can offer control over size, rigidity, drug encapsulation, and storage stability in biomedical applications, but their design and optimization remain largely empirical.

NP-cell adhesion is also expected to be influenced by the state of the cytoskeleton or the extracellular matrix, which (in the current study) is represented as the pinning of a deformable membrane with cytoskeletal proteins that bind the cell membrane to the substrate or extracellular matrix ${ }^{23}$. An earlier study demonstrates that the pinning induces confinement in the membrane undulations and, as a result, leads to novel and non-trivial membrane topographies, which could impact the binding and the uptake of the NP to the cell membrane ${ }^{24}$. The uptake of functionalized NPs into the cell membrane is accompanied by membrane shape evolution, including the formation of buds, invaginations, or protrusions, that are later pinched off the membrane as vesicles through protein-mediated scission. NPs enclosed in such vesicles can be transported to different locations inside the cell, commonly referred to as intracellular 
transport. Biophysical and thermodynamic principles governing NP adhesion and uptake in response to both NP and membrane mechanical properties are addressed in this study.

Although it is clear that the stiffness of NPs functionalized with targeting antibodies has significant effects on their interaction with cells, relatively little is known about the role of NP flexibility on drug delivery. This effect governed by mechanics could be crucial to the design of NPs for targeting to diseased tissues. In this context, few studies have investigated the role that NP stiffness plays in NP interactions with diseased endothelial cells and tissue targeting behavior 25-29. While the NGP construct has been physically characterized and realized as a good vehicle for drug delivery in vivo in mice models ${ }^{30}$, its optimization for targeting specific tissues is far from clinical translation. These data underscore the need to clarify the unique mechanisms through which deformable NPs may impart benefits and opportunities for engineering the biodistribution and tissue targeting.

Quantitative characterization of mechanisms modulating NGP adhesion to the host cell membrane requires a model that accurately estimates the adhesion affinity from the underlying receptor-ligand binding parameters. In this context, we have recently developed a statistical mechanics based computational framework for multivalent adhesion of functionalized rigid, rigid-tethered, and flexible NPs to a membrane surface that takes into account mechanical properties of both the NP and the cell surfaces as well as the biophysical factors of membrane anchorage 21,24 . We have also proposed new thermodynamic methods to compute entropyenthalpy compensation for the related problem of binding affinity of NPs to the cell surface ${ }^{21}$. We bring these novel computational techniques to the current study and address central questions regarding the roles of NP composition from very soft, deformable NPs to rigid spheres, 
effect of tethers, the crowding of NPs at the membrane surface, and the cell membrane properties such as membrane tension, and cytoskeletal interactions. We aim to shed light on the potential of NPs' structure and mechanics to control the binding and ultimately the uptake of the NPs, therefore opening up avenues for rationally engineering and fine-tuning of new systems in targeted drug delivery.

\section{Results}

We consider three different types of NPs in this study: flexible, rigid, and rigid-tethered NPs (Fig.

1a). The flexible NPs represent lysozyme-core/dextran-shell polymer NPs and are modeled as star polymers. The stiffness of the flexible NP is engineered by physically crosslinking the polymer chains to create an NP microstructure. Experimentally, we vary the stiffness of the flexible NP from $0.43 \mathrm{kPa}$ to $15.02 \mathrm{kPa}$; the corresponding NP models were developed in our earlier work, and the structures, given in Fig $1 b$, are referred to as Model 1 to Model $5^{16}$. The rigid NP corresponds to a ligand-coated spherical particle, and in rigid-tethered NP, the ligands are attached to the rigid NP via polymer tethers with variable molecular weight. Model 6 and Model 7 in Fig. 1b correspond to the rigid and rigid-tethered NPs, respectively; see methods for details of the NP models.

The equilibrium conformations of a cell membrane are described by the parameters that govern the physicochemical properties in our model: namely, the bending rigidity $k$, excess area $A_{\text {ex }}$,

and the presence of membrane cytoskeleton-linker-proteins, $N_{\text {pin }}{ }^{24}$. The membrane bending rigidity is taken to be $20 k_{B} T$. The dimensionless quantity $A_{e x}$, defined as $A_{e x}=\frac{A-A_{p}}{A}$, is the available excess area in the surface and is a representative of tension experienced by the cell 
membrane. Here, $A$ is the total curvilinear area, and $A_{p}$ is the projected area of the equilibrated membrane patch. We obtain the membrane configurations for different $A_{e x}$ by keeping $A_{p}$ fixed and varying $A$. The range of $A_{e x}$ explored here is $6 \%-34 \%$, which was noted for most endothelial cells ${ }^{31}$. The equilibrium conformations of the membranes are also determined by the state of cytoskeletal pinning and adhesive interactions between the membrane and extracellular matrix. The presence of these pinning or adhesion sites, their mobility, and bindingunbinding dynamics limit the out-of-plane fluctuations of the membrane and induces protrusions or invaginations in the membrane ${ }^{24}$. To understand the role of such membrane adhesions, we introduce pinning sites at randomly selected discrete points on the membrane and vary the density of pinning $\left(N_{p i n}\right)$.

\section{Effect of NP stiffness and membrane tension on NP avidity to the cell surface}

We begin our discussion by the avidity distribution of the multivalent NPs. The equilibrium conformations and fluctuations of both NPs and cell membranes are quantified by recording: (i) the probability distribution of multivalent binding interactions between the ligands on the NP and the receptors expressed on the cell surface; and (ii) the adhesion free energy computed by including both the enthalpy gain, that enhances binding, and the entropy loss, that inhibits binding of NPs.

\section{Roles of membrane excess area and NP stiffness on the multivalency of binding}

To evaluate the binding multivalency of ligand-functionalized flexible/rigid/rigid-tethered NPs, we computed the probability distribution of the number of simultaneous binding interactions 
between the ligand-receptor pairs (Fig. 2a,b and supplementary information, Movies S1-S5). Here we demonstrate that flexible NPs exhibit maximal binding multivalency to the membrane in an excess area independent manner. In the case of rigid NPs, the number of bound ligand-receptor pairs are very low, and the multivalency does not show a significant increase with $A_{e x}$. Compared to rigid and flexible NPs, rigid-tethered NPs exhibit a broad multivalency distribution $P(m)$ that is bimodal. In this case, $A_{e x}$ plays a crucial role in binding, showing higher multivalency at higher $A_{e x}$. Our results show that the formation of receptor-ligand bonds in the case of rigid-tethered NPs induces noticeable membrane invaginations (insets of Fig. $2 \mathrm{a}, \mathrm{b}$ ) at the site of binding and nearly internalizes the NP at high $A_{e x}$. The membrane deformations at a higher excess area allow the rigid-tethered NPs to gain a high number of bound ligand-receptor pairs, as high as the bond formation observed for flexible NPs. Representative snapshots of flexible NPs (inset of Fig. 2 a,b) suggests that a pancake-like configuration is often adopted for almost all stiffness of NPs to maximize the surface coverage and therefore the binding enhancement. The probability of multivalent binding interactions for flexible NPs and their snapshots with different stiffness are provided in Supplementary Fig. 1.

\section{Free energy analysis}

To predict the overall binding affinity (also referred to as the binding avidity) of the NPs, we quantify the equilibrium state of a ligand-coated NP bound to a substrate in terms of the losses in the configurational entropies and the gains in the binding enthalpies in the system. Our earlier studies have shown that multivalent interactions between the NP and the cell surface are subject to entropy-enthalpy compensation associated with receptor-ligand translation, NP flexibility, and 
membrane undulations ${ }^{16,32,33}$. The free energy landscape for NP binding is analyzed by explicitly computing the total free energy of binding as described in the Supplementary Discussion, also see ${ }^{34,35}$.

Free energy analysis for different type NPs is shown in Fig. 2 c-h. We observe a significant enthalpy-entropy compensation in the binding of flexible NPs to the membrane, and it depends on the stiffness of the flexible NP and $A_{\text {ex }}$ of the membrane. That is, flexible NPs experience a significant loss of configurational entropy due to polymer flexibility (Fig. 2e), an ingredient specific to the flexible NPs. This loss of configurational entropy restricts conformational fluctuations, but results in a higher multivalency of binding (Fig. 2a,b), and a favorable free energy of binding (Fig. 2c,f). This trade-off is unique to flexible NPs and is absent in both rigid and rigidtethered NPs.

When a rigid-tethered NP is bound to a membrane, the enthalpy gain between complementary receptor-ligand pairs significantly exceeds the loss of total entropy (Fig. 2 c,d), resulting in a stronger binding free energy (Fig. 2c). Moreover, upon adhesion, the rigid-tethered NPs draw the available excess membrane area to form invaginations that wrap around NPs, resulting in a substantial increase in enthalpy of binding (Fig. 2d). In effect, the results suggest stronger binding free energy for rigid-tethered NPs.

Furthermore, the multivalent binding interactions between a rigid NP and a cell membrane (both excess areas) results in a small enthalpic gain (Fig, $2 \mathrm{~d}$ ) that is completely offset by the equivalent loss in entropy (Fig. 2e), resulting in no net gain in total affinity (Fig. 2c). Even though the binding entropy and enthalpy of a receptor-ligand pair for a rigid NP (Fig. 2 g,h) compare in magnitude 
with those of flexible NPs, the lower number of bound receptor-ligand pairs in the case of rigid NP contributes to the weakening of their total free energy of binding (Fig. 2f).

Owing to competing enthalpic and entropic terms, there is no obvious systematic trend in how the total free energy of a flexible NP interacting with the cell membrane depends on the engineered changes in NP flexibility due to crosslinking (Fig. 2c). This observation agrees with those reported in earlier experimental studies ${ }^{36}$ for in-vivo retention of ICAM-targeted flexible NPs in mouse lungs (inset of Fig. $2 \mathrm{c}$ and Supplementary Fig. 7). It must be noted that the in-vivo model 1 corresponds to the uncrosslinked flexible NP and the in-vivo model 4 corresponds to the flexible NP with the highest crosslinking density. The total binding strength (Fig. $2 \mathrm{c}$ ) and the binding strength per receptor-ligand bond (Fig. 2f) are nearly constant for uncrosslinked (i.e., Model 1), intermediate crosslinked (i.e., Models 2 and 3), and highest crosslinked (i.e., Model 5) NPs, at both the membrane excess areas. Interestingly, our study demonstrates that Model 4 of flexible NP experiences the lowest binding avidity, and interpreting this result requires a further analysis of the individual energy components, see below.

We report the individual configurational entropies associated with receptors, NP, and membrane, as well as the enthalpic gain due to the interaction between receptors and ligands, and bead-to-bead interactions of flexible NP with different stiffness in Supplementary Table 1. It is evident from the table that the entropic and enthalpic terms governing receptor-ligand interactions have the most dominant effects on the magnitude of the binding strength, but they both are invariant concerning the NP stiffness. However, the enthalpic term associated with NP flexibility (bead-bead interaction) for model 4 indicates that internal strain energy in the polymer conformations is being traded for increased multivalency, which impacts the total overall binding 
strength, and this effect is strongly dependent on the membrane excess area. Comparing across all models of flexible NP, the bead-to-bead NP interactions show a favorable enthalpy gain as the NP stiffness is increased from model 1 to model 3 and model 4 to model 5 . This trend, however, becomes reversed as the stiffness of NP is increased from model 3 to model 4 for the reasons noted above regarding model 4 . Interestingly, the entropy change in membrane undulation plays a role, although not a significant one in determining the overall binding strength.

\section{Role of crowding environment on the binding of NP}

While the findings elucidated so far, have shown the dominant roles of a single NP's type and stiffness and the characteristic deformation of a membrane (i.e., $A_{\text {ex }}$ ) on their binding mechanism to a flexible membrane, a comprehensive understanding of the process requires the consideration of NP crowding. The major challenge is posed by the cellular environment, which may be densely crowded by other macromolecules and proteins, making the diffusion and binding of an NP difficult. It was usually thought that such a dense environment hinders an NP's transport looking for its binding site on the cell surface. However, it is known that the crowders seem to facilitate the transport and search process of the proteins and enhance their binding interactions ${ }^{37}$. The effects of crowding can be direct (e.g., steric hindrance or increase in effective concentration) or indirect (e.g., competition for available receptors for binding, membrane mediated interactions between two NPs adjacently bound), and the positive or negative effects can only be quantified through accounting for such interactions directly in the model.

We hypothesize that the stiffness of NPs, type of NPs, and membrane $A_{e x}$ impose barriers to the binding of multiple NPs to the cell surface. To test this hypothesis, we evaluate the effects of NP 
density (as crowding agents) on the NP-membrane conformational change and binding interactions by increasing the concentration of NPs from one to eight NPs, as depicted in Fig. 3 a,b and Supplementary Fig. 2 (See also supplementary information, Movies S6-S11). As noted, it is not necessary that the involved NPs interact with each other, such as through specific binding or association. Rather, NPs influence each other indirectly through membrane deformations caused by their adhesion. Figs. 3 a,b (Models 1 and 2) and Supplementary Fig. 2 a,b for the flexible NPs shows that when a relatively small number of NPs are present, a pancake-like configuration is still adopted to maximize the surface coverage and, therefore, resulting in enhanced multivalency. With an increase in the number of flexible NPs, the surface gets more crowded, and the NP chains tend to inter-digitate, resulting in a brush-like configuration. Looking at the snapshots of rigid NPs (Fig. 3a, b Model 6 and Supplementary Fig. 2 c,d), the number of bound receptor-ligand pairs per NP does not change by increasing the number of NPs, and the interacting pairs do not induce a conformational change at the binding site.

Though an increasing number of NPs did not alter membrane conformations significantly for both flexible and rigid NPs (Fig. 3a, b Model 1-6), the adhesion of rigid-tethered NPs (Fig. 3a, b Model 7 and Supplementary Fig. 2 e,f) is impacted by crowding. As the available excess area of the membrane and number receptors are shared by multiple NPs, each NP makes smaller membrane deformations than the single rigid-tethered NP case.

The free energy analysis of multiple NPs is shown in Fig. 3c-h. The increased density of NPs does not significantly influence the entropy-enthalpy compensation of rigid NPs, and they have the lowest binding strength out of all the three types of NPs (Fig. 3c,f). For flexible and rigid-tethered NPs, though we have seen a significant enthalpy gain over entropy loss in the case of single NPs, 
the crowding of NPs results in weaker binding energy per NP (Fig. 3c,f). Compared with Model 1 and Model 5 of a flexible NP, upon the presence of crowding agents, Model 5 of flexible NPs shows a relatively stronger binding avidity than Model 1 (Supplementary Fig. 3). These results further depict that the overall binding avidity per NP and receptor-ligand bond (Fig. $3 c, f)$ for Model 5 is about the same for the rigid-tethered NPs. Our results and analysis suggest that an increase in NP density can result in a weaker binding affinity for flexible and rigid-tethered NPs, whereas the affinity is independent of density for rigid-NPs. Therefore, the stiffness of flexible NPs manifests as an important design factor for the enhanced binding avidity of flexible NPs in crowded environments.

\section{Effect of membrane morphologies in NP adhesion and uptake}

The adhesion proteins, membrane-cytoskeleton linker proteins, and membrane-ECM attachment proteins play a significant role in defining cell membranes' morphology. The pinning of the membrane due to such proteins not only restricts membrane undulations but induce protrusions and invaginations ${ }^{24}$. The equilibrium conformations of a membrane patch under the influence of such interactions as a function of $A_{e x}$ and pinning density $N_{p}$ is given in Supplementary Fig. 4. Here we focus on the adhesion of different types of NPs on pinninginduced membrane morphologies. Fig. 4 shows the snapshot of bound NPs on the membrane for low, intermediate, and high excess areas when $N_{p}=0.05,0.1$ (See supplementary information, Movies S12-S14 for conformational dynamics).

Comparing our results of adhesion without pinning to that with pinning, we find that both the membrane conformations (Fig 4a-c) and the multivalency distribution (Supplementary Fig. 5) 
show that the binding of NPs on the membrane is more sensitive to $A_{\text {ex }}$ than to the density of pinning sites. For low $A_{e x}$ (Fig. 4a), the pinning-induced membrane conformations remain unaltered upon binding for all type NPs except for rigid-tethered NPs. In rigid-tethered NPs, the pinning and NP-adhesion act cooperatively such that the pinning-induced invaginations coincide with the membrane area that wraps the NPs. Besides, the pinning changes the bimodal distribution of rigid-tethered NPs into unimodal (Supplementary Fig. 5). This change in binding interactions is due to the ironing out of membrane undulations by pinning sites that allow maximum membrane area for wrapping the NPs and much more homogeneous binding conformations across the replicates.

The effect of pinning is more prominent upon an increase in the membrane excess area (Fig. 4 b,c) for all type NPs. In Fig. 4c, we observe that the pinning-induced deformed membrane topographies enable adhesion-induced invaginations for the rigid NPs and full wrapping of rigidtethered NPs. Additionally, Model 1 of flexible NP accesses the ridges whereas Model 5 shows binding on protrusions and invaginations.

Free energy analysis of the membrane-bound NPs in the presence of pinning sites is shown in Fig. 4; see also Supplementary Fig. 6. Similar to the previous cases, rigid NPs exhibit the lowest binding avidity. At low $A_{\text {ex }}$ of the membrane (Fig. 4a), the binding avidity of flexible NPs is comparable in magnitude to rigid-tethered NPs. As the membrane $A_{\text {ex }}$ falls in the intermediate region (Fig. 4 b), the rigid-tethered NP promotes stronger avidity for both pinning densities compared to its flexible counterpart. However, as the membrane $A_{\text {ex }}$ increases, the rigidtethered NP has higher binding avidity only for smaller pinning densities. As the pinning density increases, both rigid-tethered and flexible NPs have comparable favorable binding energy. 
The combined effects of membrane undulation and binding interactions of ligand-receptor pairs facilitate and permit access of rigid-tethered NPs to the membrane protrusions/buds, and wrapping at the large excess area and for both pinning densities (snapshot of Fig. 4c). Therefore, the propensity for uptake of the rigid-tethered NP is not hampered by the membrane pinning. Unlike both rigid and rigid-tethered NPs, which seem to be insensitive to the change of the pinning density at both low and intermediate $A_{e x}$ of the membrane, the presence of pinning sites results in a different outcome for the binding strength of flexible NPs (Fig. 4 a,b). At a pinning density of 0.05 , the overall energy of binding is nearly invariant for both Model 1 and Model 5 , a behavior also observed when there are no pinning sites at the membrane surface. As the pinning density increases to 0.1 (Fig. 4 a,b), the out-of-plane fluctuations of the membrane are noticeably restricted by the cytoskeletal pinning sites, making Model 5 suffer from lower enthalpy gain and Model 1 benefits from lower entropy loss. The result of this enthalpy-entropy compensation promotes stronger avidity for Model 1. At high $A_{e x}$ of the membrane (Fig. 4c), the membrane fluctuations are high enough not to get restricted by the pinning, resulting in a stronger binding avidity for Model 1 than Model 5, see also supplementary Fig. 6 .

We did not observe a complete or even partial enveloping of the particles by the membrane except for the case of rigid-tethered NP. However, our results suggest that while model 5 may be more susceptible to cytoskeletal mediated uptake (actin dependent uptake) owing to its localization on sites of protrusion and invagination, model 1 is preferentially adhered pinned membrane surface owing to the stronger avidity. 


\section{Effect of curvature inducing proteins at the site of NP uptake in the cell surface}

Curvature effective proteins are known to play a significant role in the adhesion and internalization of nanocarriers and viruses; clathrin-mediated endocytosis being the best-known example ${ }^{38-40}$. Previous studies of internalization of NPs in the presence of curvature-inducing proteins explored the effect of shape and aspect ratio in the uptake of particles ${ }^{15}$. Here we focus on the effect of NP flexibility and membrane deformation when the receptor-ligand binding interaction between the NP and the membrane initiates a protein coat assembly at the surface. In our model system, the assembled protein coats induce a curvature $0.06 / \mathrm{nm}$ on the membrane, a choice motivated by and justified in a previous study reporting clathrin-mediated endocytosis 40.

The representative conformations of the membrane-NP system and the multivalency for low and intermediate excess areas are shown in Fig. 5. The presence of curvature-inducing proteins causes a significant increase in multivalency only in the case of rigid-tethered NPs, for both values of $A_{\text {ex }}$. In this case, the proteins stabilize a higher curvature around the NPs, allowing for more binding events. The cooperative action of proteins and the adhesion interaction of ligands and receptors results in the uptake of rigid-tethered NPs at high $A_{\text {ex }}$, whereas the available area limits the low Aex case due to the higher tension experienced by the membrane. For flexible NPs, the binding multivalency has already been saturated, and hence the additional protein-induced curvature does not affect the multivalency significantly. However, the binding-induced assembly of proteins causes a large membrane deformation, a curvature high enough to cover and uptake the NP at high $A_{\text {ex }}$. In the case of rigid NPs, as the multivalency is lower, the observed effect of protein coat is negligible. 


\section{Comparison with experimental data}

Computational methodologies based on Monte Carlo (MC) or/and coarse-grained molecular dynamics (CGMD) protocols for the binding of functionalized NPs to both functionalized compliant and non-compliant surfaces have been developed and extensively validated in previous works ${ }^{16,31,32,34,41}$. A summary of published reports is highlighted in Supplementary Table 2 which have reported experimental validation of the rigid and flexible models. Therefore, we present the validation of the rigid-tethered NP binding below.

As explained in the Methods, we have performed the endothelial cell culture experiment for the binding of antibody-PEGylated gold NPs, having distinct molecular weights, to the Cho-ICAM cells. The average size of coated gold NPs, determined by the dynamic light scattering, for PEG with molecular weights of 2,5 , and $10 \mathrm{kDa}$ is $57.74,64.6$, and $76.2 \mathrm{~nm}$, respectively. We plot the number of bound gold NPs against the corresponding total amount added in Supplementary Fig. 8a.

In our recent studies, we looked at the role of NP size on the binding affinity of functionalized rigid-tethered NPs to the cell surface. This phenomenon was demonstrated in Fig.9(b) of ${ }^{32}$ by measuring the equilibrium multivalency for NPs having five different sizes $R_{\text {rigid-tethered }}=$ $40,150,250,350$, and $500 \mathrm{~nm}$, and two different spring constants $k_{\text {rigid-tethered }}=0.1$ and $1 \mathrm{~N} / \mathrm{m}$. The corresponding total number of ligands on the NPs were selected to be $N_{l}=$ $162,1458,4050,7938$, and 16200 , such that NPs have a uniform ligand density of $14 \%$. We utilize that information and plot the number of bound ligands versus the total number of ligands added per NP in Supplementary Fig. 8c. 
For the comparison purpose, we choose antibody-PEGylated gold NPs with a molecular weight of $5 \mathrm{kDa}$ (i.e., $R_{\text {rigid-tethered }} \cong 40 \mathrm{~nm}$ ) (Supplementary Fig. 8b). In Supplementary Fig. 8d we compare the number of bound ligands against the total number of ligands added. This comparison result is normalized by the average number of ligands and is in excellent agreement.

\section{Discussion and Conclusions}

Using a biophysically inspired coarse-grained subcellular model and thermodynamic analysis, we demonstrate that numerous factors such as NP type, stiffness, and concentration, state of the membrane cytoskeleton, and curvature remodeling proteins can selectively induce cellular targeting and uptake of functionalized NPs. We report tunable exploration of binding and uptake of three distinct NPs that differ in type and rigidity (flexible, rigid, and rigid-tethered) but are otherwise similar in size and ligand surface density.

Based on our results, the fact that the flexible NPs can spread and flatten on the target surface and maximize multivalent interactions may have advantages in affinity targeting over the rigidtethered NPs. However, we also find that the collaborative action of curvature-inducing proteins and the receptor-ligand binding interactions facilitate complete membrane wrapping (enveloping) of flexible and rigid-tethered NPs, which can be exploited for tuning NP uptake by cells. Our study reveals that the adhesion of a flexible NP depends non-trivially on engineered changes in NP stiffness. Therefore, the rational engineering of NP flexibility requires understanding the dependence of the underlying enthalpic and entropic terms that dominate. The dominant effects are context-specific (i.e., depend on NP type, cytoskeletal state, and on the membrane excess area), and the crowding density of NPs. We discuss below the specific learnings 
from our exploration of the individual effects and provide conclusions that can directly benefit in the rational design of flexible NPs in applications warranting the optimization of binding avidity, binding selectivity (specificity), and cellular uptake.

Non intuitive dependence of NP binding on stiffness: Our results show that the NP strain energy exhibits a maximum at intermediate stiffness (model 4), see bead-bead interaction energy in Table S1, and remains low for low as well as high stiffness NP causing the trends to be non-systematic. NPs exceeding this intermediate stiffness threshold have different binding thermodynamics compared to NPs with stiffness below the threshold. Significant enthalpy-entropy associated with multivalent binding, receptor diffusion, and membrane undulations (especially amidst crowding) make the predictions context specific and dependent on membrane properties (tension) and cytoskeletal state.

Tension or Excess Area Sensors: In the absence of tethers or if tethers mediating ligand-receptor interactions are of comparable stiffness to the NP, then there is minimal effect of the membrane excess area on binding avidity (models 1-3 and 6). If the tether is softer than NP stiffness (models 4,5 , and 7) then there is a definite dependence of binding on membrane excess area. In this latter case, membrane /cytoskeletal tension can be exploited as a switch to regulate NP binding. Cortical tension, therefore, impacts NP binding for some (but not all) NP stiffness, which can be exploited for targeting NPs to specific cell types.

Effect of crowding: The effect of increasing NP concentration is more pronounced in the presence of flexible or rigid-tethered NPs as they exhibit significantly greater binding avidity relative to their rigid counterpart. However, since the available excess area of the membrane and number receptors are shared by multiple flexible or rigid-tethered NPs, each NP experiences weaker binding energy than the single NP case. 
All of the NP models we have investigated with the exception of the rigid NP (model 6) are limited by the availability of receptors on the membrane surface for binding owing to the large multivalency. Saturating multivalency in crowded conditions would imply a local density of receptors that is greater than the average surface density, which translates to an entropic penalty, a scenario that manifests as a strong dependence of crowding on the binding avidity.

In addition, we have uncovered the effect of crowding is further mediated by the membrane excess area for models 5, 7 but not for models 1,6 (among the four models investigated for this compound effect of crowding and Aex). We conclude that for models of intermediate stiffness (neither too rigid nor too flexible), membrane mediated interactions further induce negative cooperativity on binding implying that the tension sensing properties of such NPs are impacted by crowding. The mechanism that mediates such an effect is also evident from our results. In this case, the local deformation fields on the membrane upon NP binding confer the negative cooperativity in binding in a crowded environment owing to the fields interacting with each other; such interactions are long ranged and hence are in play even under modest crowding scenarios ${ }^{42}$ However, the negative cooperativity does not manifest for NP of low stiffness (model 1) or rigid NP (model 6), where the induced deformation field is weak. ${ }^{21}$

Effect of cytoskeleton-induced heterogeneity: Under the influence of pinning interactions due to the cytoskeleton, the adhesion behavior is strikingly different among the various of NPs. Both flexible and rigid-tethered NPs in the presence or absence of pinning sites exhibit favorable binding avidity to the cell surface. However, the pinning sites on the membrane limit the out-ofplane fluctuations of the membrane. We find that the membrane tension and the pinning density exquisitely influence how the stiffness of flexible NPs can alter the energy of adhesion of flexible NPs. The flexible NPs (model 1) bind throughout the surface whereas the highly crosslinked 
(model 5) bind preferentially on protrusions and invaginations. The rigid NP (model 6) induces curvature amidst pinning owing to the fact that the membrane excess area is spatially nonuniformly distributed amidst pinning, while the rigid-tethered NP (model 7) continues to display full wrapping compared to the other NP models which don't show wrapping. Based on the change in the binding free energy with membrane excess area, we conclude that all NP models can sense a change in pinning density at low membrane excess area and can be sensors of the cytoskeletal state under these conditions.

Degree of crosslinking in flexible NP can be used to engineer the sensing of the cytoskeletal state. Our results suggest that uncrosslinked NP (model1) shows higher selectivity in binding to membrane with greater pinning density while the opposite is true when the degree of crosslinking was increased (model 5).

NP Uptake through curvature effector proteins: The protein coat assemblies consisting of curvature effector proteins (introduced in our model as curvature fields) promote the formation of membrane invaginations and uptake. The observed multivalency distribution and membrane conformations suggest that flexible and rigid-tethered NPs will have low barriers for NP uptake when curvature effector proteins are recruited to the site of NP binding.

However, a possible scenario could involve the curvature effector proteins relying on the induced curvature upon NP binding to be recruited through a curvature sensing mechanism. ${ }^{43,44}$ Under such a mechanism, only NP with high stiffness (model 5) or rigid-tethered NP (model 7) would be able to recruit the curvature effector proteins at the binding site and therefore benefit from the additional extrinsically (i.e. curvature not coupled to multivalent adhesion) induced-curvature for NP uptake. However, alternative mechanisms regulating access to spatially clustered receptors or noncanonical internalization routes can favor the uptake of other NP types. ${ }^{22,45,46}$ 
Conclusion: Our computational analysis shows that tuning NP stiffness impacts enthalpic and entropic contributions to multivalent adhesion and uptake through both cytoskeletal dependent and curvature dependent mechanisms. The results also show cellular targeting and uptake can be rationally engineered by the tuning of both NP and cell mechanical properties. These results collectively suggest that flexibility of NPs can be leveraged to engineer essential drug delivery properties of NPs, and NP stiffness can be best utilized in tandem with modifications of other physical and chemical parameters to create more advanced NP delivery systems.

\section{Methods: Computational models, Endothelial cell culture, Binding of ligand-coated NPs}

Computational Methods: We have developed a statistical mechanics-based computational framework to predict and assess the binding affinity of a ligand-coated flexible NP and their accessibility to undulating cell membranes by considering the equilibrium number of bound receptor-ligand pairs ${ }^{21}$. The conformational state of the NP interacting with the cell membrane is defined in terms of interaction potentials and is evolved through the Metropolis Monte Carlo (MC) approach coupled with the Brownian dynamics method to sample the microstates of the NP-membrane system at thermal equilibrium. A detailed description of the computational model and analysis, and a summary of the detailed parameter set are made available in the Supplementary Discussion and Supplementary Table 3. The simulations are performed in a $500 \times 500 \times 620 \mathrm{~nm}^{3}$ simulation box with periodic boundary conditions along the membrane surface. We first equilibrate the membrane and the NP independently for $5 \times 10^{7} \mathrm{MCs}$. We then place the NP near the membrane surface and allow the system to relax for $5 \times 10^{8}$ MCs before collecting the data. Four independent simulation trajectories are generated for each set of 
conditions. To estimate the error in the calculated quantities, we calculate the average value for each independent trajectory and then calculate the standard deviation over the four ensembles. The computations for a typical trajectory noted above require three CPU-weeks on a single core of a $2.7 \mathrm{GHz}$ processor.

Rigid polystyrene and Flexible nanogel particles (NGPs): Methods for preparation, characterization, and assaying of binding, uptake, and targeting of rigid and flexible particles were described in earlier works $19,20,22,41$ and will not be repeated here. We discuss the protocols for rigid-tethered particles because experimental results for such particles are presented for the first time here.

Preparation of $\mathbf{5 0 ~} \mathbf{n m}$ gold nanoparticles (AuNPs): To prepare $50 \mathrm{~nm}$ AuNPs, first, $2 \mathrm{~mL}$ of $1 \%$ $\mathrm{HAuCl} 4$ solution was added in $200 \mathrm{~mL}$ of DI water in a conical ask. Next, $6 \mathrm{ml}$ of $15 \mathrm{~nm}$ seed particles solution was added to the solution while stirring. Finally, $440 \mu L 1 \%$ tri-sodium citrate and $2 \mathrm{~mL}$ of 0:03 $\mathrm{M}$ hydroquinone were added, respectively. The solution was left an hour on a stir plate to observe color transition.

Expression and purification of protein Z L17 mutations: The PSTEPL plasmid containing the cloned protein Z L17 sequence and the pEVOL- pBpF plasmid (Addgene.org) were co-transformed into the T7 Expression Crystal Competent Cells (New England Bio-labs). Bacterial starter cultures were then grown in $2 \mathrm{~mL} \mathrm{LB}+100 \mu \mathrm{g} / \mathrm{mL}$ ampicillin $+25 \mu \mathrm{g} / \mathrm{mL}$ chloramphenicol at $37^{\circ} \mathrm{C}$ in a shaker overnight. Starter cultures were added at a 1:1000 dilution to Autoinduction Media LB Broth Base Including Trace Elements (Formedium), $100 \mu \mathrm{g} / \mathrm{mL}$ ampicillin, and $25 \mu \mathrm{g} / \mathrm{mL}$ chloramphenicol. For BPA incorporation, L-benzoylphenylalanine (Bachem, King of Prussia, PA) was added into the culture for a final concentration of $500 \mathrm{M}$, and arabinose was added into the 
culture to a final concentration of $0: 1 \%$ to begin the inductions of the pEVOL plasmid. Protein ZL17 was expressed at $37^{\circ} \mathrm{C}$ in a shaker for $24 \mathrm{~h}$ and pelleted by centrifugation.

For lysis, cell pellets were resuspended in B-PER (Thermo Fisher Scientific) with $200 \mu \mathrm{g} / \mathrm{mL}$ lysozyme, $4 \mu \mathrm{g} / \mathrm{mL}$ DNAsel, and 1 complete Mini EDTA-free Protease Inhibitor Tablet (Roche). Resuspended pellets were incubated at room temperature for $30 \mathrm{~min}$ and then put in the freezer to increase the lysis efficiency. The lysates clarified by centrifugation were incubated with cobalt resin (TALON Metal Affinity Resin, Clontech, $1.2 \mathrm{~mL}$ per $100 \mathrm{~mL}$ expression culture) for $30 \mathrm{~min}$ at room temperature for binding. Following the incubation, resin was washed by PBS. For labeling protein Z L17, $500 \mu L \mathrm{PBS}+50 \mathrm{M} \mathrm{CaCl} 2+200 \mathrm{M}$ GGGSK(DBCO)NH2 peptide (NEO Scientific) pH 7.4 was added and inoculated for $4-6$ hours at $37^{\circ} \mathrm{C}$. The product was purified from access labeling reagent using a $3 \mathrm{kDa}$ molecular weight cut-off (MWCO) filter (Amicon Ultra, Millipore, Temecula, CA). Purified DBCO labeled protein Z L17 was checked by SDS-PAGE gel, and concentration was quantified by BCA assay (Pierce, Rockford, IL).

Copper-free Click Chemistry: For copper-free click conjugation, three reactions were prepared with different molecular weight Thiol-PEG-Azide (2 kDa, $5 \mathrm{kDa}$, and $10 \mathrm{kDa})$. DBCO labelled protein $Z$, and different molecular weight Thiol-PEG-Azide were mixed with a 1:3 molar ratio overnight at room temperature. The next day, reactions were used for antibody cross-linking.

Preparation of Antibody-PEG-Thiol: Protein Z clicked PEG-Thiol mixes were incubated with R6.5 at the molar ratio of $1 \operatorname{lgG}$ to 8 Protein Z- PEG Thiol in a clear centrifuge tube on ice for 2 hours with $365 \mathrm{~nm}$ UV light using a UVP CL-1000L UV crosslinker (Upland, CA). Crosslinked products were purified from access proteins and PEG-Thiol mix using 100 kDa molecular weight cut-off (MWCO) filter (Amicon Ultra, Millipore, Temecula, CA), and then they were analyzed directly 
using SDS-PAGE electrophoresis. For reducing SDS-PAGE, samples were boiled for 5 min with SDSPAGE loading buffer (Bio-rad, Hercules, CA) containing 1:20 dilution of beta-mercaptoethanol (Bio-rad). The samples were run for $30 \mathrm{~min}$ at constant 180 Volts. The gels were stained for visualization by using the SimplyBlue Coomassie stain (Invitrogen).

Antibody-PEG-Thiol radiolabeling: Antibody-PEG-Thiol was radiolabeled with $N a^{125} I$ using Pierce lodination Beads. The reaction was carried out for 15 min at rt. Free $125 \mathrm{I}$ was removed by Quick Spin Protein Columns (G-25 Sephadex, Roche Applied Science, Indianapolis, IN). Gold radiolabeling: Direct labeling of gold nanoparticles was carried out through chemisorption of iodine-125 ${ }^{47}$. Gold nanoparticles were radiolabeled with $N a^{125} I$ using Pierce lodination Beads. The reaction was carried out for $15 \mathrm{~min}$ at $\mathrm{rt}$. Free $125 \mathrm{I}$ was removed by three washes in distilled $\mathrm{H}_{2} \mathrm{O}$ and centrifugation at $3000 \times \mathrm{G}$ for 30min. Thin-layer chromatography (TLC) was performed using alumina-backed silica gel to determine the percent of free $125 \mathrm{I}$. The mobile phase consisted of $75 \%$ Methanol and $25 \%$ Ammonium acetate. TLC plates were analyzed using a Typhoon 9410 Molecular Imager (GE Healthcare Life Sciences, Pittsburgh, PA). The radiochemical purity was then determined as the area of the free 125 I divided by the area sum of the total.

Antibody-PEGylation of AuNPs: Antibody-PEG-thiol moieties were conjugated at different number ratios to gold nanoparticles in PBS. Thiolated polyethylene glycol (PEG-SH) was used to coat any remaining bare gold surfaces to reduce nonspecific interactions. The particles were incubated for $15 \mathrm{~min}$ under agitation on a shaker. The antibody-conjugated particles were washed three times in PBS with 1\%BSA/0.1\% Tween and centrifugation at $3000 \times \mathrm{G}$ for $30 \mathrm{~min}$. 
Binding of targeted gold NPs: Cho-ICAM cells were plated on 24 well plates (Corning Inc., Corning, NY) and grown to confluence $\left(105 \mathrm{cell} / \mathrm{cm}^{2}\right)$. The radiolabeled gold NPs were added to the corresponding cells and incubated for $1 \mathrm{~h}$ at $4^{\circ} \mathrm{C}$. After incubation, the cells were washed three times with ice-cold Hanks Balanced Salt Solution (HBSS, Corning Cellgro, Manassas, VA), and then lysed with lysis buffer (1\% Triton X-100, $1 \mathrm{M} \mathrm{NaOH})$. The lysates were measured using Wallac 1470 Wizard $^{T M}$ gamma counter (Gaithersburg, MD). The bound radioactive gold NPs were plotted against the corresponding total amount added in Supplementary Fig. 8.

Characterization: Synthesized and coated AuNPs were characterized by UV-absorption spectroscopy (Varian, 100 Bio), plasma optical emission spectroscopy (ICP-OES) (Spectro Genesis, GMBH), DLS (Malvern, Zetasizer, Nano-ZS), TEM (Tecnai, T12) analysis.

\section{Acknowledgment}

We acknowledge financial support from the National Institutes of Health through grants CA227550, CA244660. This work used the Extreme Science and Engineering Discovery Environment (XSEDE), supported by National Science Foundation grant number ACl-1548562.

\section{References:}

1 Rai, R., Alwani, S. \& Badea, I. Polymeric Nanoparticles in Gene Therapy: New Avenues of Design and Optimization for Delivery Applications. Polymers (Basel) 11, doi:10.3390/polym11040745 (2019).

2 Chen, J., Guo, Z., Tian, H. \& Chen, X. Production and clinical development of nanoparticles for gene delivery. Mol Ther Methods Clin Dev 3, 16023, doi:10.1038/mtm.2016.23 (2016). 
3 Gong, N., Sheppard, N. C., Billingsley, M. M., June, C. H. \& Mitchell, M. J. Nanomaterials for T-cell cancer immunotherapy. Nat Nanotechnol 16, 25-36, doi:10.1038/s41565-02000822-y (2021).

4 Yu, Q., Othman, S., Dasgupta, S., Auth, T. \& Gompper, G. Nanoparticle wrapping at small non-spherical vesicles: curvatures at play. Nanoscale 10, 6445-6458, doi:10.1039/C7NR08856F (2018).

5 Zhu, X. et al. Multiplex reverse transcription loop-mediated isothermal amplification combined with nanoparticle-based lateral flow biosensor for the diagnosis of COVID-19. Biosens Bioelectron 166, 112437, doi:10.1016/j.bios.2020.112437 (2020).

6 Ghaffari, M. et al. An overview of the use of biomaterials, nanotechnology, and stem cells for detection and treatment of COVID-19: towards a framework to address future global pandemics. Emergent Mater, 1-16, doi:10.1007/s42247-020-00143-9 (2021).

7 Mitchell, M. J. et al. Engineering precision nanoparticles for drug delivery. Nat Rev Drug Discov 20, 101-124, doi:10.1038/s41573-020-0090-8 (2021).

8 Dubacheva, G. V., Curk, T., Frenkel, D. \& Richter, R. P. Multivalent Recognition at Fluid Surfaces: The Interplay of Receptor Clustering and Superselectivity. J Am Chem Soc 141, 2577-2588, doi:10.1021/jacs.8b12553 (2019).

9 Mammen, M., Choi, S. K. \& Whitesides, G. M. Polyvalent Interactions in Biological Systems: Implications for Design and Use of Multivalent Ligands and Inhibitors. Angew Chem Int Ed Engl 37, 2754-2794, doi:10.1002/(SICI)15213773(19981102)37:20<2754::AID-ANIE2754>3.0.CO;2-3 (1998). 
10 Collins, B. E. \& Paulson, J. C. Cell surface biology mediated by low affinity multivalent protein-glycan interactions. Curr Opin Chem Biol 8, 617-625, doi:10.1016/j.cbpa.2004.10.004 (2004).

11 Albertazzi, L. et al. Spatiotemporal control and superselectivity in supramolecular polymers using multivalency. Proc Natl Acad Sci U S A 110, 12203-12208, doi:10.1073/pnas.1303109110 (2013).

12 Tito, N. B. \& Frenkel, D. Optimizing the Selectivity of Surface-Adsorbing Multivalent Polymers. Macromolecules 47, 7496-7509, doi:10.1021/ma5014918 (2014).

13 Tito, N. B., Angioletti-Uberti, S. \& Frenkel, D. Communication: Simple approach for calculating the binding free energy of a multivalent particle. J Chem Phys 144, 161101, doi:10.1063/1.4948257 (2016).

14 Vcha, R., Martinez-Veracoechea, F. J. \& Frenkel, D. Intracellular release of endocytosed nanoparticles upon a change of ligand-receptor interaction. ACS Nano 6, 10598--10605 (2012).

15 Deng, H., Dutta, P. \& Liu, J. Stochastic modeling of nanoparticle internalization and expulsion through receptor-mediated transcytosis. Nanoscale 11, 11227-11235, doi:10.1039/c9nr02710f (2019).

16 Farokhirad, S. et al. Stiffness can mediate balance between hydrodynamic forces and avidity to impact the targeting of flexible polymeric nanoparticles in flow. Nanoscale 11, 6916-6928, doi:10.1039/C8NR09594A (2019). 
17 Shen, Z. et al. Polymer stiffness governs template mediated self-assembly of liposomelike nanoparticles: simulation, theory and experiment. Nanoscale 11, 20179-20193, doi:10.1039/c9nr07063j (2019).

18 Li, B. \& Abel, S. M. Shaping membrane vesicles by adsorption of a semiflexible polymer. Soft Matter 14, 185-193, doi:10.1039/c7sm01751k (2018).

19 Carme Coll Ferrer, M., Sobolewski, P., Composto, R. J. \& Eckmann, D. M. Cellular Uptake and Intracellular Cargo Release From Dextran Based Nanogel Drug Carriers. Journal of nanotechnology in engineering and medicine 4, 110021-110028, doi:10.1115/1.4023246 (2013).

20 Coll Ferrer, M. C., Dastgheyb, S., Hickok, N. J., Eckmann, D. M. \& Composto, R. J. Designing nanogel carriers for antibacterial applications. Acta Biomater 10, 2105-2111, doi:10.1016/j.actbio.2014.01.009 (2014).

21 Farokhirad, S., Bradley, R. P. \& Radhakrishnan, R. Thermodynamic analysis of multivalent binding of functionalized nanoparticles to membrane surface reveals the importance of membrane entropy and nanoparticle entropy in adhesion of flexible nanoparticles. Soft Matter 15, 9271-9286, doi:10.1039/C9SM01653H (2019).

22 Myerson, J. W. et al. Flexible Nanoparticles Reach Sterically Obscured Endothelial Targets Inaccessible to Rigid Nanoparticles. Adv Mater, e1802373, doi:10.1002/adma.201802373 (2018).

23 Anitei, M. \& Hoflack, B. Bridging membrane and cytoskeleton dynamics in the secretory and endocytic pathways. Nat Cell Biol 14, 11-19, doi:10.1038/ncb2409 (2012). 
24 Kandy, S. K. \& Radhakrishnan, R. Emergent membrane morphologies in relaxed and tense membranes in presence of reversible adhesive pinning interactions. Physical Biology 16, 066011, doi:10.1088/1478-3975/ab48d5 (2019).

25 Merkel, T. J. et al. Using mechanobiological mimicry of red blood cells to extend circulation times of hydrogel microparticles. Proceedings of the National Academy of Sciences 108, 586, doi:10.1073/pnas.1010013108 (2011).

26 Sun, H. et al. The role of capsule stiffness on cellular processing. Chemical Science 6, 35053514, doi:10.1039/C5SC00416K (2015).

27 Anselmo, A. C. et al. Platelet-like Nanoparticles: Mimicking Shape, Flexibility, and Surface Biology of Platelets To Target Vascular Injuries. ACS Nano 8, 11243-11253, doi:10.1021/nn503732m (2014).

28 Anselmo, A. C. et al. Elasticity of Nanoparticles Influences Their Blood Circulation, Phagocytosis, Endocytosis, and Targeting. ACS Nano 9, 3169-3177, doi:10.1021/acsnano.5b00147 (2015).

29 Zhang, L. et al. Microfluidic Synthesis of Hybrid Nanoparticles with Controlled Lipid Layers: Understanding Flexibility-Regulated Cell-Nanoparticle Interaction. ACS Nano 9, 99129921, doi:10.1021/acsnano.5b05792 (2015).

30 Ferrer, M. C. et al. Icam-1 targeted nanogels loaded with dexamethasone alleviate pulmonary inflammation. PLoS One 9, e102329, doi:10.1371/journal.pone.0102329 (2014). 
31 Ramakrishnan, N. et al. Biophysically inspired model for functionalized nanocarrier adhesion to cell surface: roles of protein expression and mechanical factors. $R$ Soc Open Sci 3, 160260, doi:10.1098/rsos.160260 (2016).

32 McKenzie, M., Ha, S. M., Rammohan, A., Radhakrishnan, R. \& Ramakrishnan, N. Multivalent Binding of a Ligand-Coated Particle: Role of Shape, Size, and Ligand Heterogeneity. Biophysical Journal 114, 1830-1846, doi:10.1016/j.bpj.2018.03.007 (2018).

33 Ramakrishnan, N., Tourdot, R. W. \& Radhakrishnan, R. Thermodynamic free energy methods to investigate shape transitions in bilayer membranes. Int J Adv Eng Sci Appl Math 8, 88-100, doi:10.1007/s12572-015-0159-5 (2016).

34 Liu, J. et al. Computational model for nanocarrier binding to endothelium validated using in vivo, in vitro, and atomic force microscopy experiments. Proc Nat Acad Sci 107, 1653016535 (2010).

35 Liu, J. et al. Multivalent Binding of Nanocarrier to Endothelial Cells under Shear Flow. Biophysical Journal 101, 319-326, doi:10.1016/j.bpj.2011.05.063 (2011).

36 Myerson, J. W. et al. Cross-linker-Modulated Nanogel Flexibility Correlates with Tunable Targeting to a Sterically Impeded Endothelial Marker. ACS Nano 13, 11409-11421, doi:10.1021/acsnano.9b04789 (2019).

37 Phillips, R., Kondev, J. \& Theriot, J. Physical biology of the cell. (Garland Science, 2009).

38 McMahon, H. T. Endocytosis: an assembly protein for clathrin cages. Current biology : $C B$ 9, R332-335 (1999). 
39 Ramanan, V. et al. Systems biology and physical biology of clathrin-mediated endocytosis. Integrative biology : quantitative biosciences from nano to macro 3, 803-815, doi:10.1039/c1ib00036e (2011).

40 Tourdot, R. W., Bradley, R. P., Ramakrishnan, N. \& Radhakrishnan, R. Multiscale computational models in physical systems biology of intracellular trafficking. IET Syst Biol 8, 198-213, doi:10.1049/iet-syb.2013.0057 (2014).

41 Zern, B. J. et al. Reduction of nanoparticle avidity enhances the selectivity of vascular targeting and PET detection of pulmonary inflammation. ACS Nano 7, 2461-2469, doi:10.1021/nn305773f (2013).

42 Yolcu, C., Haussman, R. C. \& Deserno, M. The Effective Field Theory approach towards membrane-mediated interactions between particles. Advances in Colloid and Interface Science 208, 89-109, doi:https://doi.org/10.1016/i.cis.2014.02.017 (2014).

43 Bradley, R. P. \& Radhakrishnan, R. Curvature-undulation coupling as a basis for curvature sensing and generation in bilayer membranes. Proc Natl Acad Sci U S A 113, E5117-5124, doi:10.1073/pnas.1605259113 (2016).

44 Tourdot, R. W., Ramakrishnan, N., Baumgart, T. \& Radhakrishnan, R. Application of a freeenergy-landscape approach to study tension-dependent bilayer tubulation mediated by curvature-inducing proteins. Phys Rev E Stat Nonlin Soft Matter Phys 92, 042715, doi:10.1103/PhysRevE.92.042715 (2015).

45 Bhowmick, T., Berk, E., Cui, X., Muzykantov, V. R. \& Muro, S. Effect of flow on endothelial endocytosis of nanocarriers targeted to ICAM-1. Journal of Controlled Release doi:10.1016/j.jconrel.2011.09.067 (2011). 
46 Muro, S. \& Muzykantov, V. R. Design Parameters Modulating Intracellular Drug Delivery: Anchoring to Specific Cellular Epitopes, Carrier Geometry, and Use of Auxiliary Pharmacological Agents. Organelle-Specific Pharmaceutical Nanotechnology, 449-474, doi:https://doi.org/10.1002/9780470875780.ch24 (2010).

47 Walsh, A. A. Chemisorption of iodine-125 to gold nanoparticles allows for real-time quantitation and potential use in nanomedicine. J Nanopart Res 19, 152, doi:10.1007/s11051-017-3840-8 (2017). 


\section{Figures}

(a)

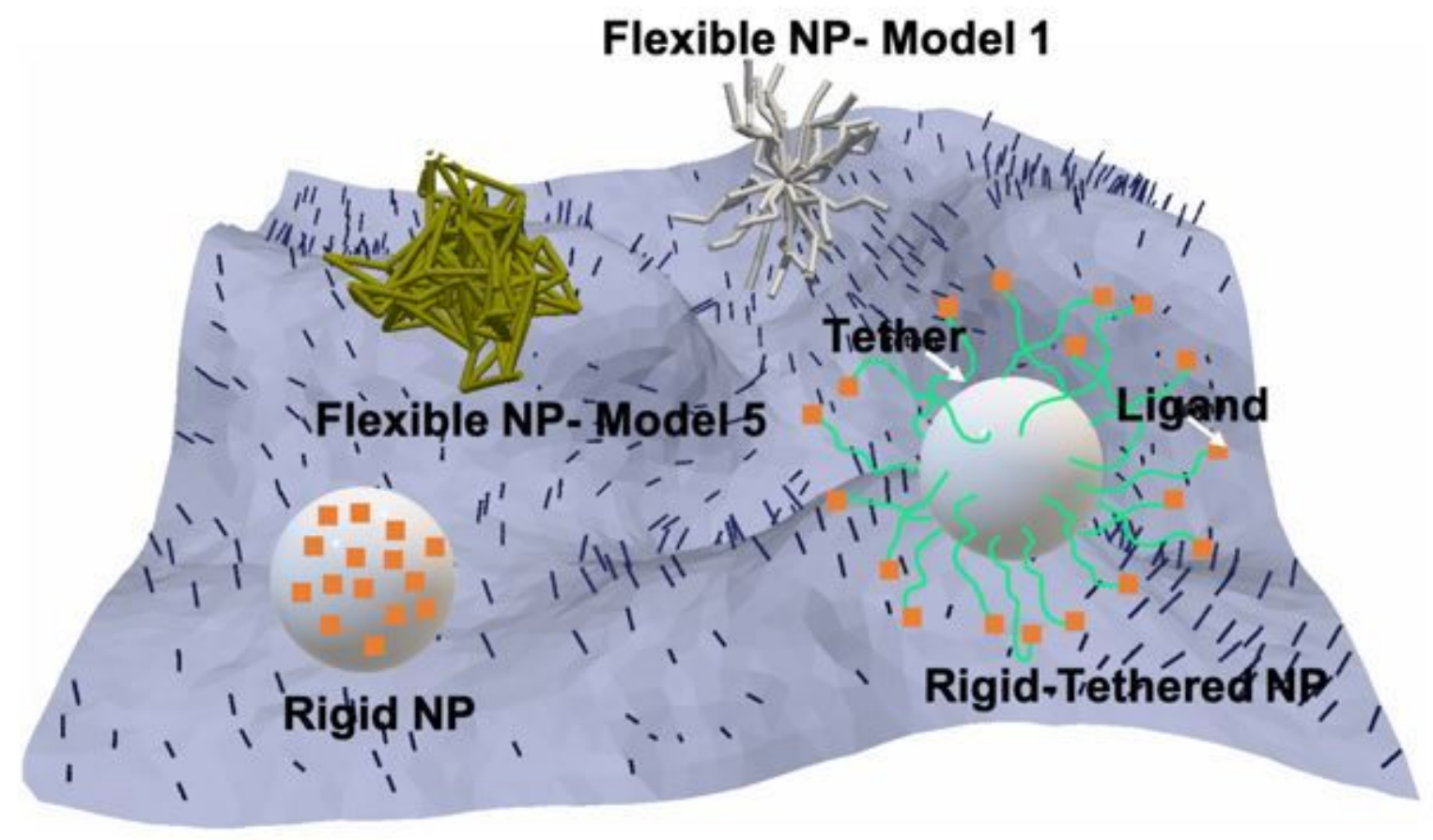

(b)

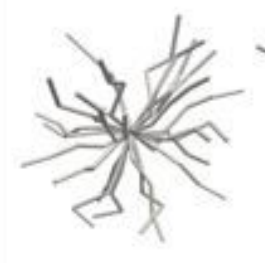

Model 1

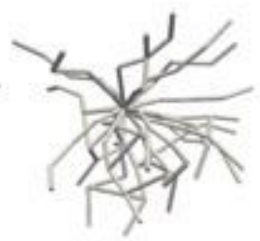

Model 2

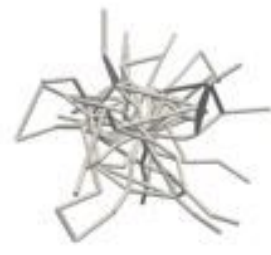

Model 3

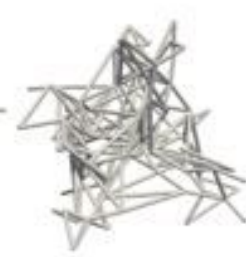

Model 4

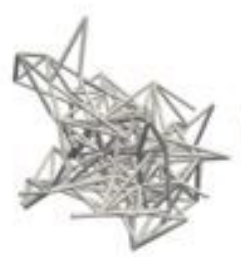

Model 5

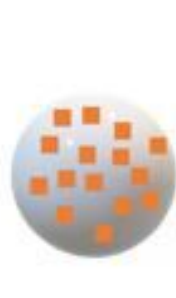

Model 6

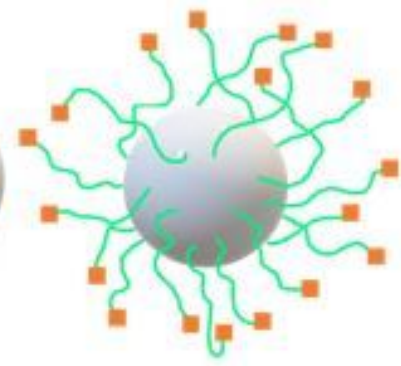

Model 7

Figure 1

Computational framework of multivalent binding of ligand-coated nanoparticle and receptors that mediate the binding. a, A representative image of membrane-NP computational model to estimate binding avidity of ligand-coated NPs to a substrate functionalized with the target receptors. b, Structures of simulated NPs. Models 1-5 are flexible NPs arranged in the increasing order of crosslinking density, Model 1 is a star polymer with zero crosslinking (i.e., elasticity modulus of $0.43 \mathrm{kPa}$ ) and Model 5 
represents a highly crosslinked NP microstructure (i.e., elasticity modulus of $15 \mathrm{kPa}$ ). With increasing crosslinking, the shape and stiffness of flexible NPs change. Model 6 and 7 represents rigid and rigidtethered NPs respectively.

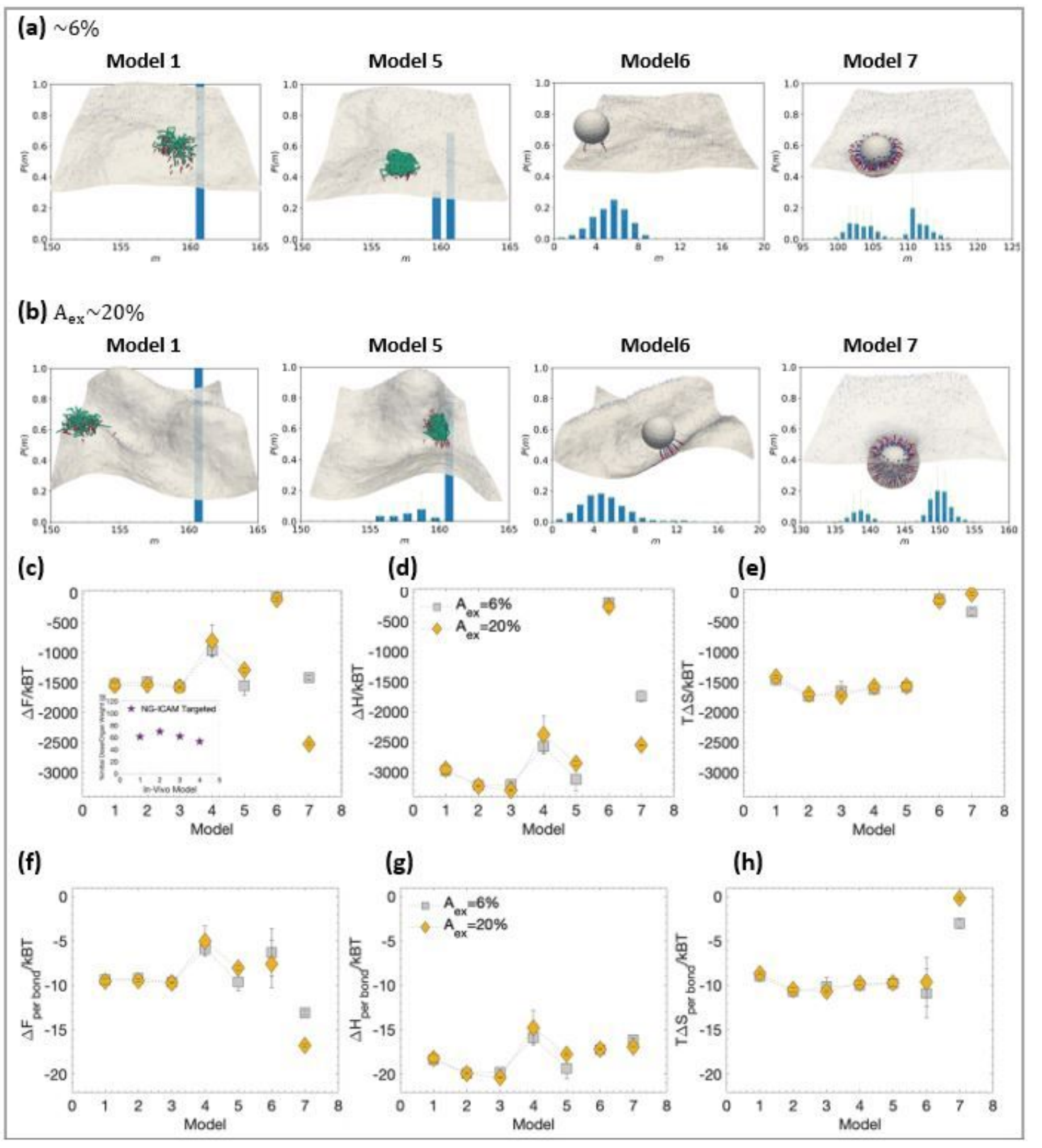

\section{Figure 2}

Binding of functionalized NPs to membrane bound ICAM receptors. a, b, Equilibrium multivalency distribution of the number of simultaneous receptor-ligand bonds. Inset: Snapshots of a flexible NP with 
different stiffness, a rigid NP, and a rigid-tethered NP, with number of antibody $=162 / \mathrm{NP}$, bound to two distinct cell membrane types, through specific, multivalent receptor-ligand interactions. c-h, Comparison of relative free energy, enthalpy, and entropy of binding for different type of NPs (c-e) and the corresponding measures per receptor-ligand bonds (f-h). Error bars of the relative energies are smaller than symbol sizes. Note: Models 1-5 correspond to flexible NPs with different stiffness and Models 6 and 7 correspond to rigid and rigid-tethered NPs, respectively. The corresponding movies for flexible NP binding to membrane can be found in the supplementary information, Movies S1-S5.

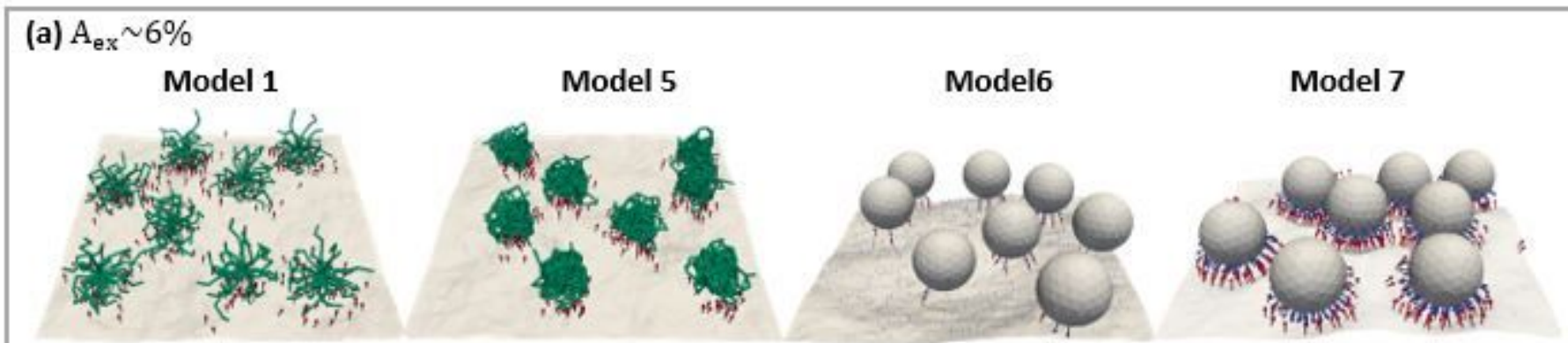

(b) $\mathrm{A}_{\mathrm{ex}} \sim 20 \%$

Model 1

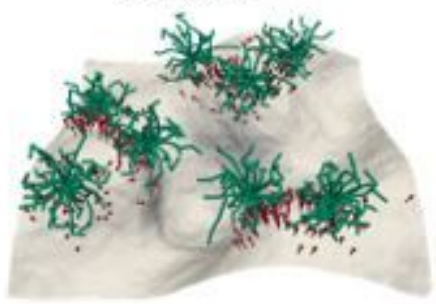

(c)

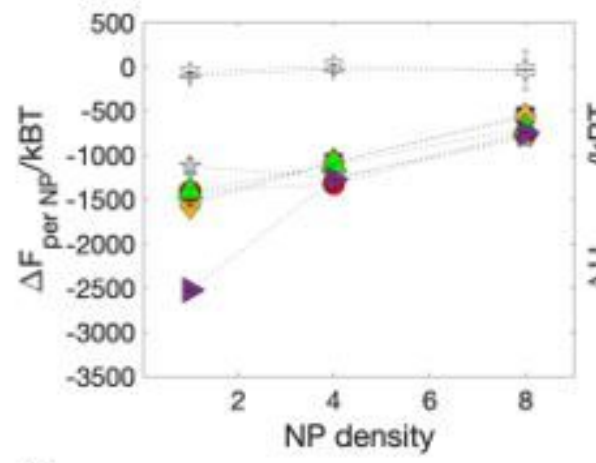

(f)

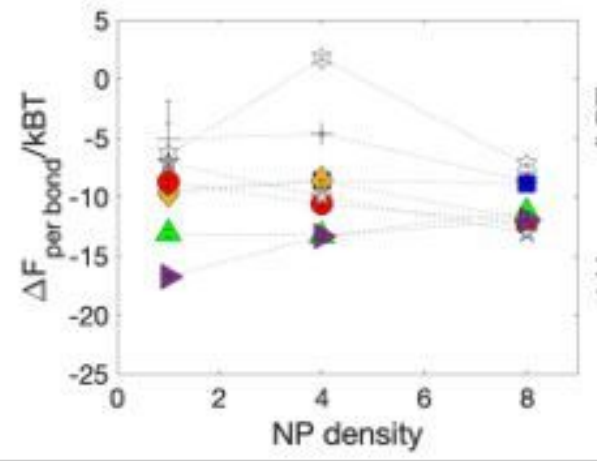

Model 5

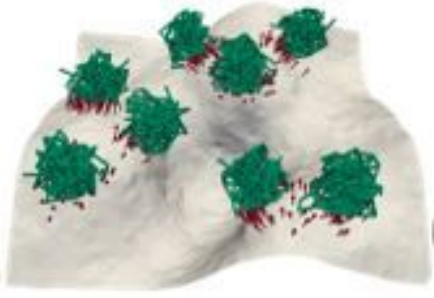

(d)

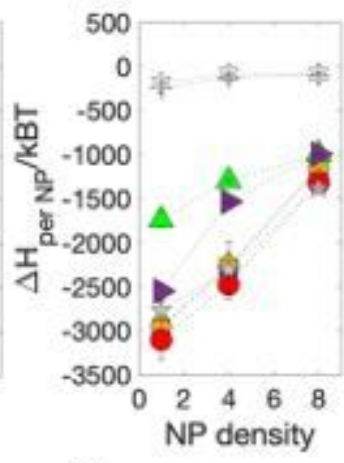

(g)

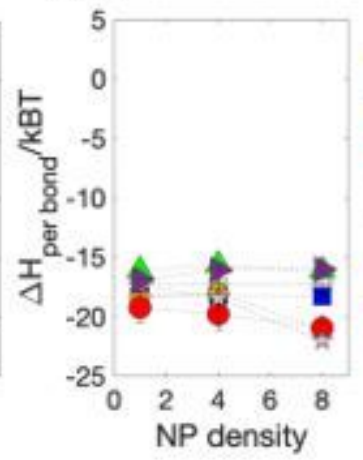

Model6

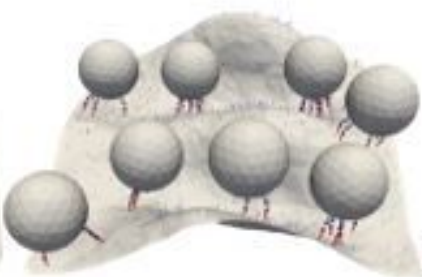

(e)

- A ex $-6 \%$-Model 1

(4) $\mathrm{A}_{\mathrm{ex}}=20 \%$-Model 1

A $A_{\text {ex }}-6 \%$-Model 5

if $A_{\text {ex }}=20 \%$-Model 5

$A_{e x}=6 \%$-Model 6

$A_{\text {ex }}=20 \%$-Model 6

A $\mathrm{A}_{\mathrm{ex}}=6 \%$-Model 7

$-A_{e x}=20 \%$-Model 7

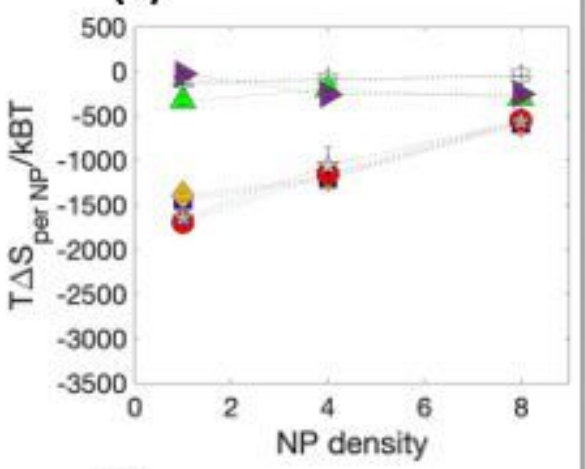

(h)

- $A_{e x}-6 \%$-Model 1

4 $A_{e x}=20 \%$-Model 1

- $A_{\mathrm{ez}}=6 \%$-Model 5

- $A_{e x}=20 \%$-Model 5

$A_{\mathrm{ex}}=6 \%$-Model 6

$A_{\mathrm{ex}}=20 \%$-Model 6

A $\quad A_{2 x}=6 \%-$ Model 7

- $A_{e x}=20 \%$-Model 7

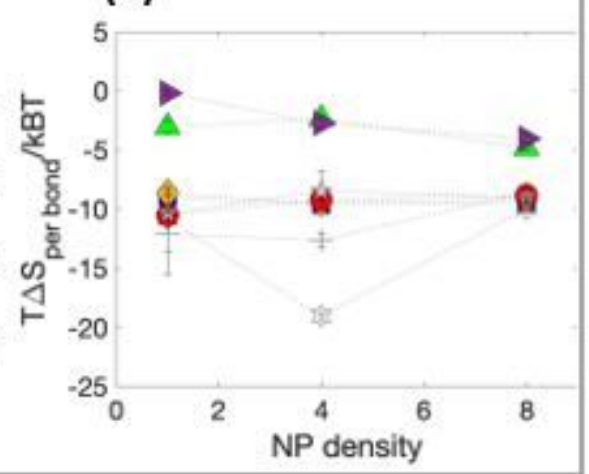

Figure 3 
Crowding of functionalized NPs bound to membrane. a, b, Snapshots of multiple flexible NPs with different stiffness, multiple rigid NPs, and multiple rigid-tethered NPs, with number of antibody $=162 / N P$, bound to two characteristically cell membrane types, having distinct excess areas Aex), through specific, multivalent receptor-ligand interactions; the corresponding movies can be found in the Supplementary Movies 6-11. c-h, Effect of crowding on the relative free energy, enthalpy, and entropy of binding. Panels ce show free energy, entropy, and enthalpy of binding per NP and panels f-h show free energy, entropy, and enthalpy of binding per receptor-ligand bond. Error bars of the relative energies are smaller than symbol sizes. Note: Models 1-5 correspond to flexible NPs with different stiffness and Models 6 and 7 correspond to rigid and rigid-tethered NPs, respectively. 


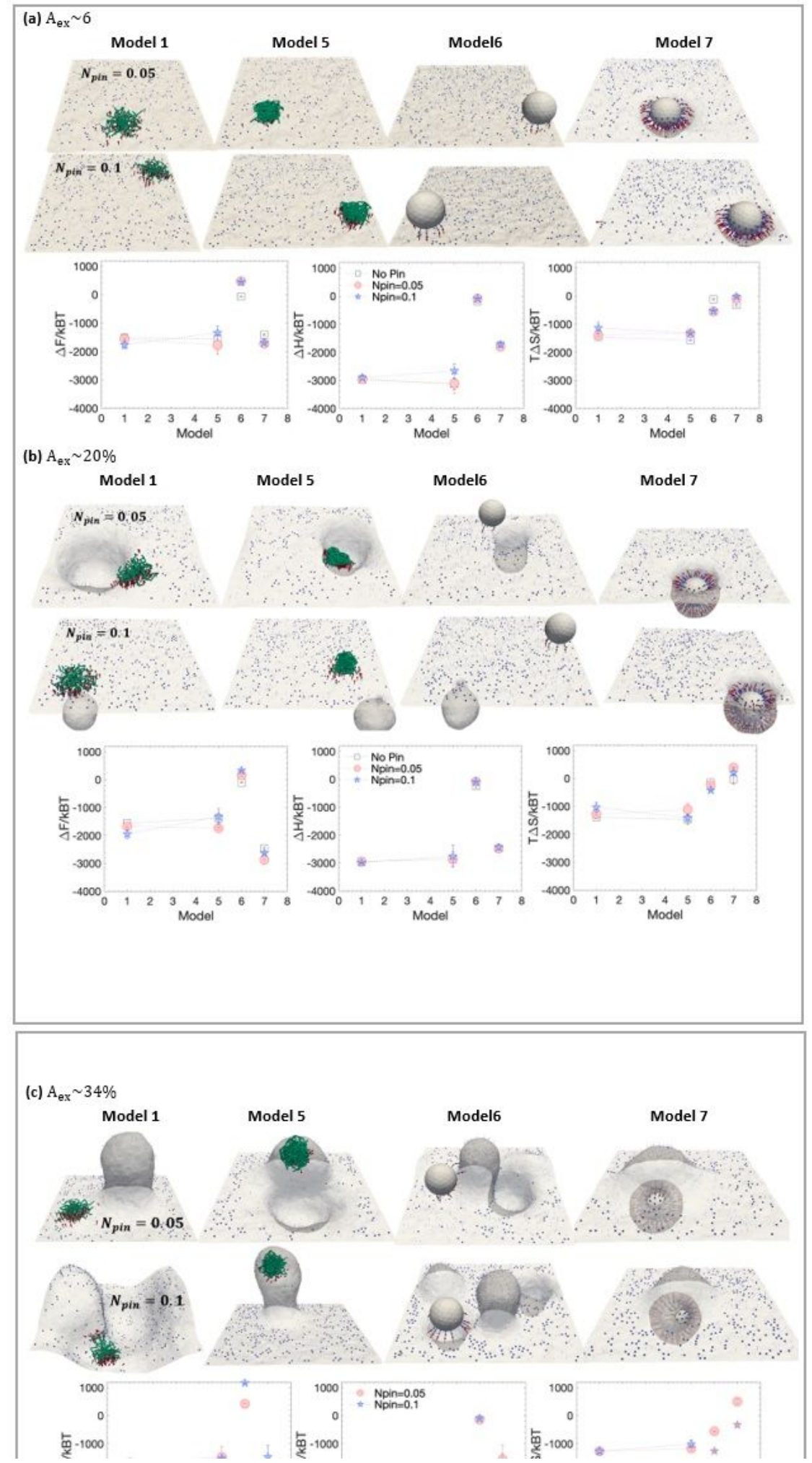

\section{Figure 4}

Adhesion of flexible, rigid, and rigid-tethered NPs in the presence of membrane cytoskeletal interactions. a-c, Relative free energy, enthalpy, and entropy of binding of NP-membrane system and their snapshots in presence of flexible, rigid, and rigid-tethered NPs, showing the effect of pining interactions at different A_ex. The blue spheres on the membrane shows the locations of the pinning sites. The corresponding 
movies for NP binding to pinned membrane can be found in the supplementary information, Movies S12S14.

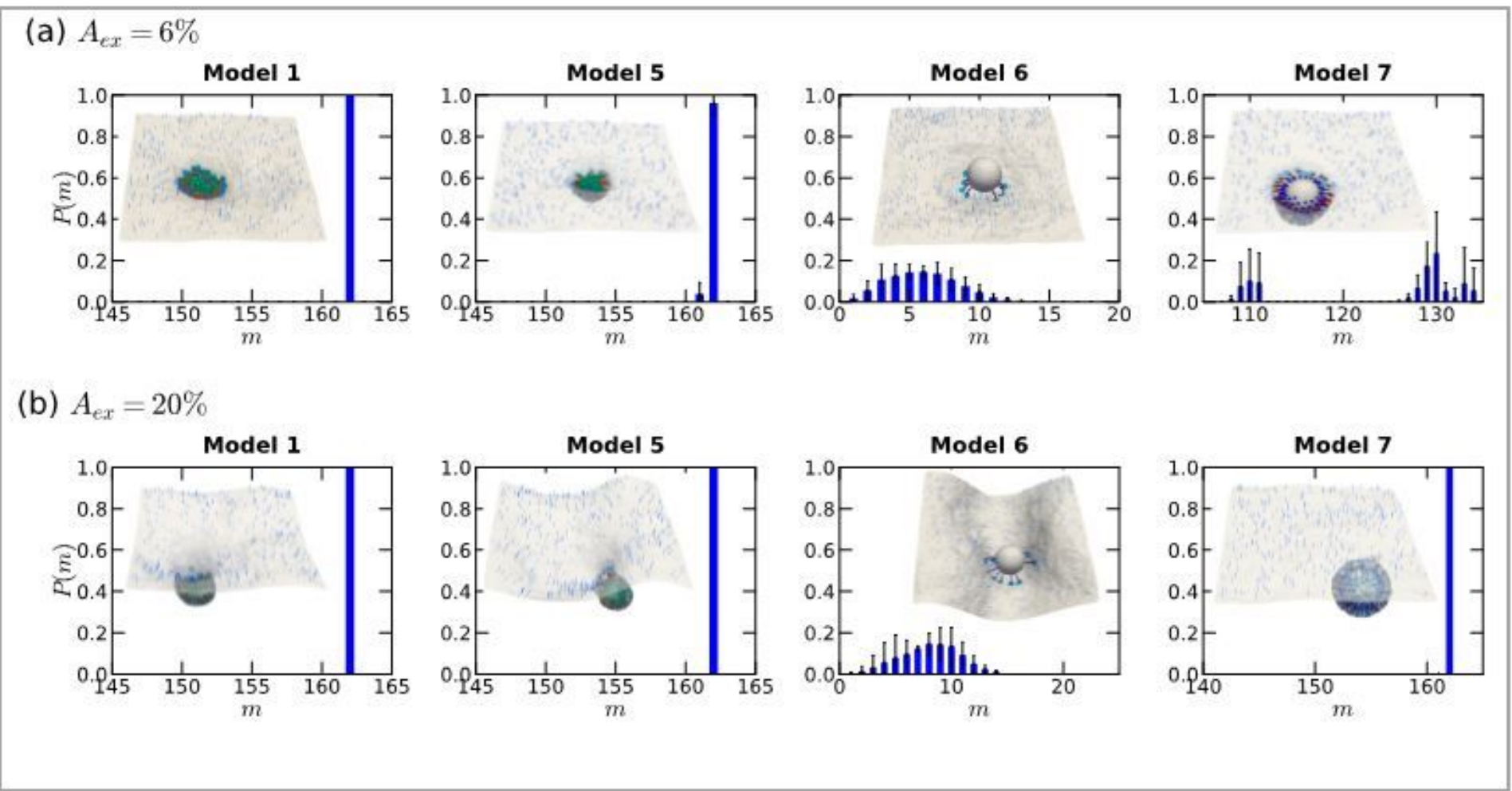

\section{Figure 5}

Curvature inducing protein. a, b, Histogram representation of multivalency and corresponding snapshots of membrane-NP system in the presence of curvature inducing proteins (shown in blue on the surface) at low and intermediate excess areas. Each receptor-ligand binding at the surface assembles a protein coat that induces a curvature $0.06 / \mathrm{nm}$.

\section{Supplementary Files}

This is a list of supplementary files associated with this preprint. Click to download.

- SIV12SF.docx

- SupplementaryMovie1.mp4

- SupplementaryMovie2.mp4

- SupplementaryMovie3.mp4

- SupplementaryMovie4.mp4

- SupplementaryMovie5.mp4

- SupplementaryMovie6.mp4

- SupplementaryMovie7.mp4

- SupplementaryMovie8.mp4 
- SupplementaryMovie9.mp4

- SupplementaryMovie10.mp4

- SupplementaryMovie11.mp4

- SupplementaryMovie12.mp4

- SupplementaryMovie13.mp4

- SupplementaryMovie14.mp4 